\title{
Deoxyribonucleic acid-based photochromic material for fast dynamic holography
}

\author{
Andrzej Miniewicz, ${ }^{\text {a) }}$ Anna Kochalska, and Jaroslaw Mysliwiec \\ Institute of Physical and Theoretical Chemistry, Wroclaw University of Technology, 50370 Wroclaw, Poland \\ Anna Samoc and Marek Samoc \\ Laser Physics Centre, Research School of Physical Sciences and Engineering, The Australian National \\ University, Canberra ACT 0200, Australia \\ James G. Grote \\ Materials and Manufacturing Directorate, U.S. Air Force Research Laboratory, Wright-Patterson Air Force \\ Base, Ohio 45433-7707
}

(Received 23 May 2007; accepted 26 June 2007; published online 27 July 2007)

\begin{abstract}
The authors report on a biopolymeric material made of deoxyribonucleic acid (DNA) complexed with the cationic surfactant cetyltrimethyl-ammonium (CTMA) and doped with the photochromic disperse red 1 dye (DR1) for dynamic holographic recording. The molar ratio of the DNA-CTMA to the dye is about 5:1. They have found that the photochromic properties of DR1 in the DNA-CTMA matrix are favorably modified in speed of response with respect to conventional polymeric matrices. Dynamic holographic gratings which were inscribed in DR1:DNA-CTMA films are characterized by switching times within a 1-10 ms range. An excellent reversibility of the recording process is reported. () 2007 American Institute of Physics. [DOI: 10.1063/1.2760169]
\end{abstract}

Photoinduced optical anisotropy (birefringence and dichroism) found in photochromic materials is a key feature for their use in such technical applications as optical storage, optical switching, and optical information processing. ${ }^{1,2}$ Optically bistable azobenzene derivatives receive much attention due to their efficient trans-cis-type light-induced reversible photoisomerization. ${ }^{3,4}$ Generally, azobenzenes exhibit three types of optical nonlinearities: the electronic, trans-cis photoisomerization, and thermal types, respectively. These properties can be suppressed or enhanced due to specific interactions of the photochromic molecules with various matrices in which they are embedded. ${ }^{5-8}$ The classic azobenzene derivative chromophore, 4-[N-ethyl$\mathrm{N}$-(2-hydroxyethyl)]-amino-4'-nitro-azobenzene known as disperse red 1 (DR1), acts as an initiator of photoinduced optical anisotropy changes in different matrices via molecular orientation due to multiple trans-cis-trans photoisomerization cycles. Here we investigate optical properties of DR1-doped deoxyribonucleic acid (DNA)-based biopolymer-a matrix, which can offer a chiral binding of the DR1 molecules to the unique double helix structure of DNA. Other known polymeric matrices differ in that respect from the reported here biopolymer. ${ }^{6,7}$

The DNA-based biopolymer used in this research was fabricated from purified salmon roe DNA, complexed with a cationic surfactant, cetyltrimethyl-ammonium (CTMA) chloride. The DNA conversion was performed according to the procedure $^{9-11}$ in which the aqueous solution of the DNA polymer (MW $8 \times 10^{6} \mathrm{~g} / \mathrm{mol}$ ) was added to an equal amount of the aqueous solution of CTMA, and the precipitate was collected by filtration under vacuum and purified by rinsing with de-ionized water. We prepared butanol solutions of DNA-CTMA and DR1 separately and mixed them to obtain $4.8 \%$ DR 1 in DNA-CTMA $w / w$ in the dry mass. The

\footnotetext{
a) Author to whom correspondence should be addressed; FAX: +48 (71)
} 3203364; electronic mail: andrzej.miniewicz@pwr.wroc.pl
DR1:DNA-CTMA solution was cast onto an indium tin oxide (ITO)-covered glass plate, dried in air and the $4 \mu \mathrm{m}$ thick film was sealed with another ITO plate in order to prevent photochemical oxidation ${ }^{12}$ and surface relief formation. An UV-vis spectrum of a $4 \mu \mathrm{m}$ thick dry film of DR1:DNACTMA is shown in Fig. 1. The absorption maximum positioned at $502 \mathrm{~nm}$ is characteristic for the DR1 dye.

Dynamic holographic grating recording (similar to the process used in optical image correlation ${ }^{13}$ ) was carried out using the degenerate two-wave mixing setup. Two equal intensity $s$-polarized beams $(488 \mathrm{~nm}$ or $514.5 \mathrm{~nm})$ from a cw $\mathrm{Ar}^{+}$laser were incident on the film. Their overlap gave rise to the intensity interference pattern with periodicity $\Lambda$ $=\lambda /\left(2 n_{g} \sin \theta\right)$, where $\lambda$ is the wavelength of laser light, $\theta$ is the incidence angle, and $n_{g}=1.515$ (at $633 \mathrm{~nm}$ ) is the refractive index of the glass. The formation of the grating was monitored through the first-order diffraction signal of selfdiffraction process. The diffraction efficiency $\eta$ of the grat-

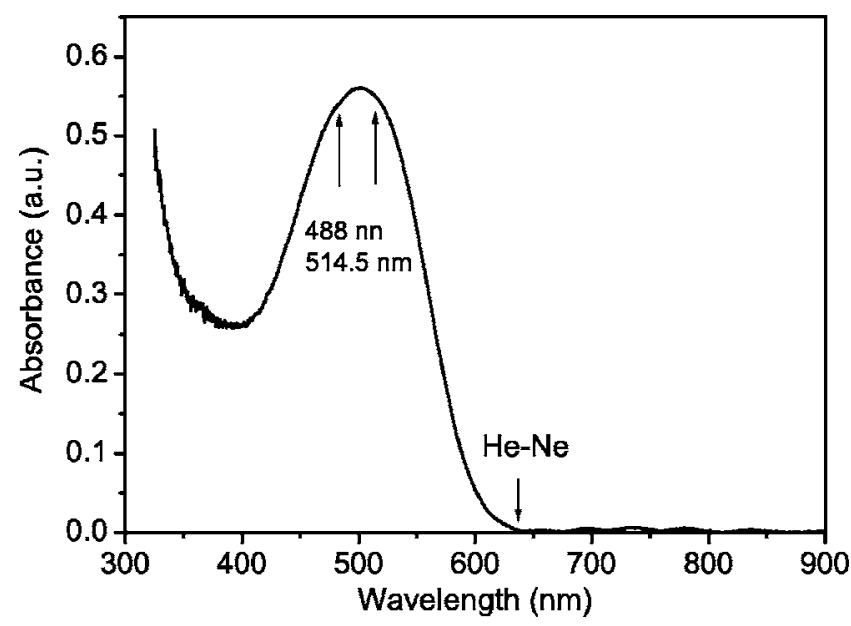

FIG. 1. Absorption spectra in UV-vis region of a $4 \mu \mathrm{m}$ thick DR1:DNACTMA film deposited on a glass substrate. 


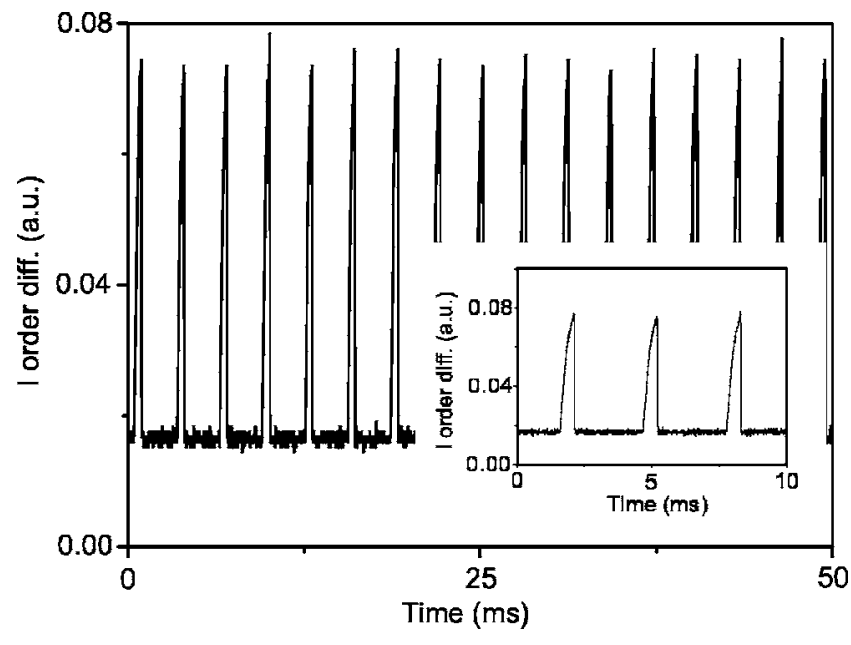

FIG. 2. Example of sequential grating recording and optical erasure in a DR1:DNA-CTMA film with $\mathrm{Ar}^{+}$laser beam $(\lambda=488 \mathrm{~nm}, 340 \mathrm{~mW}$ of total laser power, $\Lambda=12.1 \mu \mathrm{m}, s-s$ recording polarization configuration).

ing was probed with a He-Ne laser beam $(632.8 \mathrm{~nm}, 5 \mathrm{~mW})$ at a near normal incidence. The diffraction efficiency $\eta$ $=I_{\text {diff }} / I_{0}$ was calculated as the ratio of the diffracted light intensity $I_{\text {diff }}$ to the intensity of the probe laser beam $I_{0}$ incident on the sample.

The suitability of materials for real-time holography can be determined with measurements of the magnitude of the diffraction efficiency and its growth and decay rates. In the measurements of the dynamics of the grating recording we used a chopper enabling measurements of the dynamics of recording and light assisted diffraction grating erasure.

An example of the sequential grating recording and optical erasure in a DR1:DNA-CTMA film with $\mathrm{Ar}^{+}$laser light $(\lambda=488 \mathrm{~nm}, \Lambda=12.1 \mu \mathrm{m}, s-s$ recording polarization configuration) is shown in Fig. 2. Using a chopper with asymmetric light opening and closing times (1:5 duty ratio) and working with the frequency of $340 \mathrm{~Hz}$ we were able to observe appearance and decay of the first-order diffraction signal. Its magnitude stayed constant over hours of exposure. No residual light diffraction was observed after $3 \mathrm{~ms}$ from the end of each recording cycle. This indicates that the duty cycle of a potential real-time holographic device designed on the basis of the presented data may exceed 340 full cycles/s.

Next, we performed systematic measurements of the time constants of the grating recording $\left(\tau_{r}\right)$ and decay $\left(\tau_{d}\right)$ as functions of the incident recording beam power. In Fig. 3 we present the dependence of the recording time constants $\tau_{r}$ measured at 514.5 and $632.8 \mathrm{~nm}$ light wavelengths and $\tau_{d}$ obtained using the $632.8 \mathrm{~nm}$ beam diffraction on input power of one of the $\mathrm{Ar}^{+}$beams. These time constants were obtained by fitting the growth and decay of the signal with a single exponential growth function. The recording time constants were significantly shorter at higher light intensities (changing from about 8 to $2 \mathrm{~ms}$ within the range of the used powers) while the decay time constant $\tau_{d}$ was only weakly dependent on the incoming light intensity (changing from 3 to $2 \mathrm{~ms}$, respectively). At the highest set power of the recording beam $(60 \mathrm{~mW})$, which corresponded to the average light intensity at the film $I_{\text {total }}=3.4 \mathrm{~W} / \mathrm{cm}^{2}$, the time constants of the process of the grating recording and decay are equal, amounting to $2 \mathrm{~ms}$.

From the measurements of the first-order diffraction power $P_{\text {diff }}$ versus the incident laser power $P_{0}$ used for the

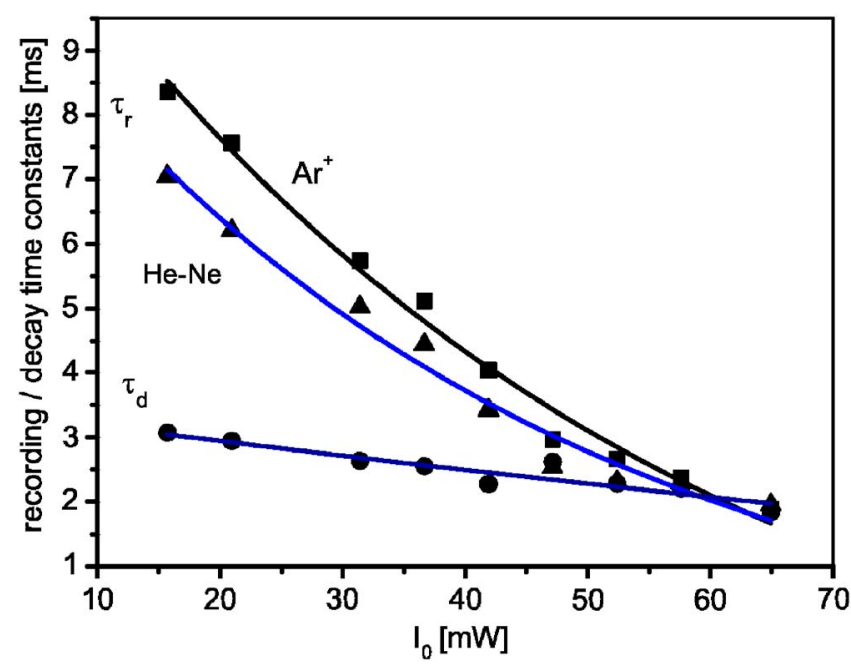

FIG. 3. Dependence of the grating formation (recording) $\tau_{r}$ and erasure $\tau_{d}$ time constants on input power of one of the $\mathrm{Ar}^{+}(514.5 \mathrm{~nm})$.

grating inscription we find a weakly superlinear dependence: $P_{\text {diff }}=A P_{0}^{\delta}$ where $\delta=1.2$. The diffraction efficiencies measured in the DR1:DNA-CTMA system are relatively small ranging from $0.0084 \%$ to $0.0016 \%$ at $I_{\text {total }}$ $=3.4-0.76 \mathrm{~W} / \mathrm{cm}^{2}$.

We performed two additional experiments in order to determine the mechanism of the formation of the laserinduced grating in the studied system. In the first experiment we checked whether a polarization grating could be formed through the light-induced alignment of trans-DR1 molecules. We inserted a $\lambda / 2$ retardation plate into one of the incoming beams to change its polarization from $s$ to $p$. In the $s-p$ configuration there is no modulation of the light intensity within the beam intersection area (in contrast to the $s$-s case), however, there is a position dependent sequence of the polarization state varying from linear through elliptical to circular. ${ }^{14}$ The $s-p$ configuration applied to polymeric systems containing DR1 molecules leads to fabrication of polarization gratings and the respective light self-diffraction. ${ }^{14}$ However, in the DR1:DNA-CTMA case we did not observe any self-diffraction for the pure $s-p$ geometry. This result indicates that there is no permanent molecular reorientation due to multiple trans-cis-trans DR1 photoisomerizations. Fast relaxation of the written gratings indicates that there is no mass transport and related surface relief gratings frequently observed in azobenzene derivative functionalized polymers. ${ }^{15,16}$

The second experiment was designed to check the option of thermal origin of the observed grating as due to light absorption by DR1 molecules the sinusoidal temperature distribution builds up. Thus local density changes due to the material thermal expansion are expected. After the light cutoff the amplitude $\Delta T$ of the temperature modulation and refractive index modulation amplitude $\Delta n \propto \Delta T$ should decay exponentially, $\Delta T=T_{0} \exp \left(-t / \tau_{\text {th }}\right)$ with the time constant $\tau_{\text {th }}$ $=\Lambda^{2} / 4 \pi^{2} \kappa$, where $\kappa$ is the thermal conduction coefficient of the material. The characteristic decay time $\tau_{\text {th }}$ should rise quadratically with the grating periodicity $\Lambda$. However, no dependence of the grating decay time constant on the grating period (within a range of periods from 3 to $17 \mu \mathrm{m}$ ) has been observed. This supports the nonthermal origin of the grating. However, the relaxation of thermal grating could be induced by a transverse heat transport through the cell surfaces ${ }^{17}$ inAlP license or copyright, see http://apl.aip.org/apl/copyright.jsp 
stead of the longitudinal diffusion in the direction of the grating vector, for which the above reasonings are appropriate.

Other experiments performed in this material [e.g., optical Kerr effect (OKE)] seem to point to a nonthermal origin of the observed grating due to noticed dependence of the OKE signal on the polarization state of the excitation beam. Therefore, the effect observed in this work can be related to light-induced spatially periodic distribution of excited-state populations when light from a fringe pattern is absorbed. This implies that the DNA-CTMA matrix does not allow for alignment of trans DR1 molecules. At present we can only speculate on the exact nature of binding of DR1 to the DNA helix. From the circular dichroism studies of Zhang et al. ${ }^{18}$ it is known that molecules of a similar dye, disperse red 13, can be oriented in DNA-CTMA in various ways (intercalated into the nanospaces between the base pairs or aligned by binding in the grooves of the DNA helix) depending on the molar ratio of DNA nucleotide base pair to the dye. In our material this molar ratio was about 5:1 (using the average formula weight of DNA-CTMA equal to $1185 \mathrm{~g} / \mathrm{mol}){ }^{19}$ Therefore, various mechanisms of binding of the dye with the DNA-based matrix are anticipated. Films of this material (4.8 wt \% DR1 in DNA-CTMA) were found to be optically isotropic $\left(n_{\mathrm{TE}}=1.5130, n_{\mathrm{TM}}=1.5117 \text { at } 632.8 \mathrm{~nm}\right)^{19}$ indicating that the dye-doped polymer chains were orientationally disordered as in neat DNA-CTMA films while the neat DNA films were birefringent. ${ }^{20}$ We expect that a selective doping with nonlinear optical dyes may bring functionalities to DNA as a photonic material.

In conclusion, the photochromic system DR1:DNACTMA is very interesting for future real-time holographic applications due to the short recovery times, good optical stability, and complete reversibility.

The authors acknowledge the Australian Research Council Discovery Project No. DP0556942, the Materials and Manufacturing Directorate Air Force Office of Scientific Research, the AOARD Grant No. 05-4010, and the Polish Ministry of Science and Higher Education Grant No.
N50713231/3302 for financial support. They also acknowledge the pioneering efforts of Naoya Ogata of the Chitose Institute of Science and Technology for providing the source of DNA. J. P. Morrall is acknowledged for carrying out the conversion of the DNA to the DNA-CTMA complex.

${ }^{1}$ J. A. Delaire and K. Nakatani, Chem. Rev. (Washington, D.C.) 100, 1817 (2000).

${ }^{2}$ J. Eickmans, T. Bieringer, S. Kostromine, H. Berneth, and R. Thoma, Jpn. J. Appl. Phys., Part 1 38, 1835 (1999).

${ }^{3}$ A. M. Makushenko, B. S. Neporent, and O. V. Stolbova, Opt. Spectrosc. 31, 295 (1971).

${ }^{4}$ G. S. Kumar and D. C. Neckers, Chem. Rev. (Washington, D.C.) 89, 1915 (1989).

${ }^{5}$ T. Saito and T. Kobayashi, Opt. Mater. (Amsterdam, Neth.) 21, 301 (2002).

${ }^{6}$ A. Natansohn and P. Rochon, Adv. Mater. (Weinheim, Ger.) 11, 1387 (1999).

${ }^{7}$ F. Chaput, J.-P. Boilot, D. Riehl, and Y. Levy, J. Sol-Gel Sci. Technol. 2, 779 (2004).

${ }^{8}$ H. Taunaumang, M. Solyga, M. O. Tija, and A. Miniewicz, Thin Solid Films 461, 316 (2004).

${ }^{9}$ L. Wang, G. Zhang, S. Horinouchi, J. Yoshida, and N. Ogata, Mol. Cryst. Liq. Cryst. Sci. Technol., Sect. B: Nonlinear Opt. 24, 63 (2000).

${ }^{10}$ L. Wang, J. Yoshida, N. Ogata, S. Sasaki, and T. Kajiyama, Chem. Mater. 13, 1273 (2001)

${ }^{11}$ E. M. Heckman, J. A. Hagen, P. P. Yaney, J. G. Grote, and F. K. Hopkins, Appl. Phys. Lett. 87, 211115 (2005).

${ }^{12}$ A. Galvan-Gonzales, M. Canva, G. I. Stegeman, L. Sukhomlinova, R. J. Twieg, K. P. Chan, T. C. Kowalczyk, and H. S. Lackritz, J. Opt. Soc. Am. B 17, 1992 (2000).

${ }^{13}$ S. Bartkiewicz, P. Sikorski, and A. Miniewicz, Opt. Lett. 23, 1769 (1998).

${ }^{14}$ P.-A. Blanche, Ph. C. Lemaire, C. Maertens, P. Dubois, and R. Jerome, Opt. Commun. 185, 1 (2000).

${ }^{15}$ J. Kumar, L. Li, X. Jiang, D.-Y. Kim, T. Lee, and S. Tripathy, Appl. Phys. Lett. 72, 2096 (1998).

${ }^{16}$ C. J. Barrett, P. L. Rochon, and A. L. Natansohn, J. Chem. Phys. 109, 1505 (1998).

${ }^{17}$ D. Grebe and R. Mcdonald, J. Phys. D 27, 567 (1994).

${ }^{18}$ G. Zhang, H. Takahashi, L. Wang, J. Yoshida, S. Kobayashi, S. Horinouchi, and N. Ogata, Proc. SPIE 4905, 375 (2002).

${ }^{19}$ A. Samoc, M. Samoc, J. G. Grote, A. Minewicz, and B. Luther-Davies, Proc. SPIE 6401, 640106 (2006).

${ }^{20}$ A. Samoc, A. Miniewicz, M. Samoc, and J. G. Grote, J. Appl. Polym. Sci. 105, 236 (2007). 